\title{
Salbutamol treatment in a patient with hyperkalaemic periodic paralysis due to a mutation in the skeletal muscle sodium channel gene (SCN4A)
}

\author{
M G Hanna, J Stewart, A H V Schapira, N W Wood, J A Morgan-Hughes, N M F Murray
}

\begin{abstract}
A 35 year old woman with clinical features of hyperkalaemic periodic paralysis confirmed on provocative exercise testing was investigated. DNA sequence analysis of the gene for the $\alpha$-subunit of the skeletal muscle voltage gated sodium channel (SCN4A) on chromosome 17 q23 identified a point mutation at nucleotide position 2188. This results in a threonine to methionine substitution at amino acid position 704. The patient was intolerant of diuretic medication but showed a striking clinical and electrophysiological improvement with salbutamol therapy. Treatment with $\beta$-adrenergic agents should be considered in patients with hyperkalaemic periodic paralysis who are intolerant of, or resistant to, diuretic medications. (F Neurol Neurosurg Psychiatry 1998;65:248-250)
\end{abstract}

Keywords: hyperkalaemic periodic paralysis; sodium channel gene (SCN4A); salbutamol

Hyperkalaemic periodic paralysis is now recognised to be one of a group of disorders known as the skeletal muscle channelopathies. ${ }^{1}$ These disorders are characterised by abnormalities of muscle fibre excitability and present with periodic paralysis or myotonia. ${ }^{1}$ A candidate gene approach has established that point mutations in the gene coding for the $\alpha$-subunit of the skeletal muscle voltage gated sodium channel on chromosome 17q23 (SCN4A) are the cause of hyperkalaemic periodic paralysis. ${ }^{2}$ This discovery has made DNA based diagnosis possible.

Therapy consists of avoiding known precipitating factors and the use of various drugs both acutely and prophylactically. Many patients respond to thiazide diuretics or acetazolamide. ${ }^{1}$ However, some patients are intolerant or resistant to such agents. We report the clinical, electrophysiological, and molecular genetic findings in a patient who responded to salbutamol.

\section{Case history}

This 35 year old white woman had experienced attacks of weakness since early childhood. Typically, attacks would be heralded by a heavy feeling in her arms and legs followed by generalised weakness which varied in severity from mild difficulty in walking to complete paralysis. The attacks rarely lasted longer than an hour and recovery was generally rapid. The most potent precipitant was strenuous exercise followed by rest. Some attacks could be aborted if she was able to move around when the heavy feelings developed. The severity of the attacks had decreased with age, but remained frequent at three or four episodes a week and interfered with her ability to work. She had not experienced any fixed weakness and attacks were not precipitated by cold. There was no history of myotonia. Treatment with hydrochlorthiazide resulted in increased frequency of attacks and a persistent complaint of lethargy. There was a family history of identical attacks in her mother, a maternal uncle, and her maternal grandmother consistent with an autosomal dominant inheritance pattern. Her mother, now aged 70, has been wheelchair bound for the last 10 years due to generalised muscle weakness. These affected relatives live abroad and were not available for examination or DNA testing.

Clinical examination of our patient disclosed eyelid myotonia but there was no evidence of myotonia elsewhere. Hip flexion was mildly weak, graded at $5-/ 5$, but there was no other weakness.

\section{Investigations}

A left quadriceps muscle biopsy done at the age of 30 was reported to be normal as were serum potassium and creatine kinase between attacks. Provocative aerobic exercise testing was performed in which her pulse rate was maintained above $160 \mathrm{bpm}$ for 30 minutes followed by strict bed rest. ${ }^{3}$ She developed an attack of weakness beginning 20 minutes after exercise in which there was marked generalised weakness and areflexia lasting for one hour. During this attack her serum potassium rose from an immediate postexercise level of $4.5 \mathrm{mmol} / 1$ to $7.4 \mathrm{mmol} / 1$, confirming the diagnosis of hyperkalaemic periodic paralysis. ECG monitoring was normal except for an occasional atrial ectopic beat. and in revised form 24

November 1997

Accepted 25 November 1997 

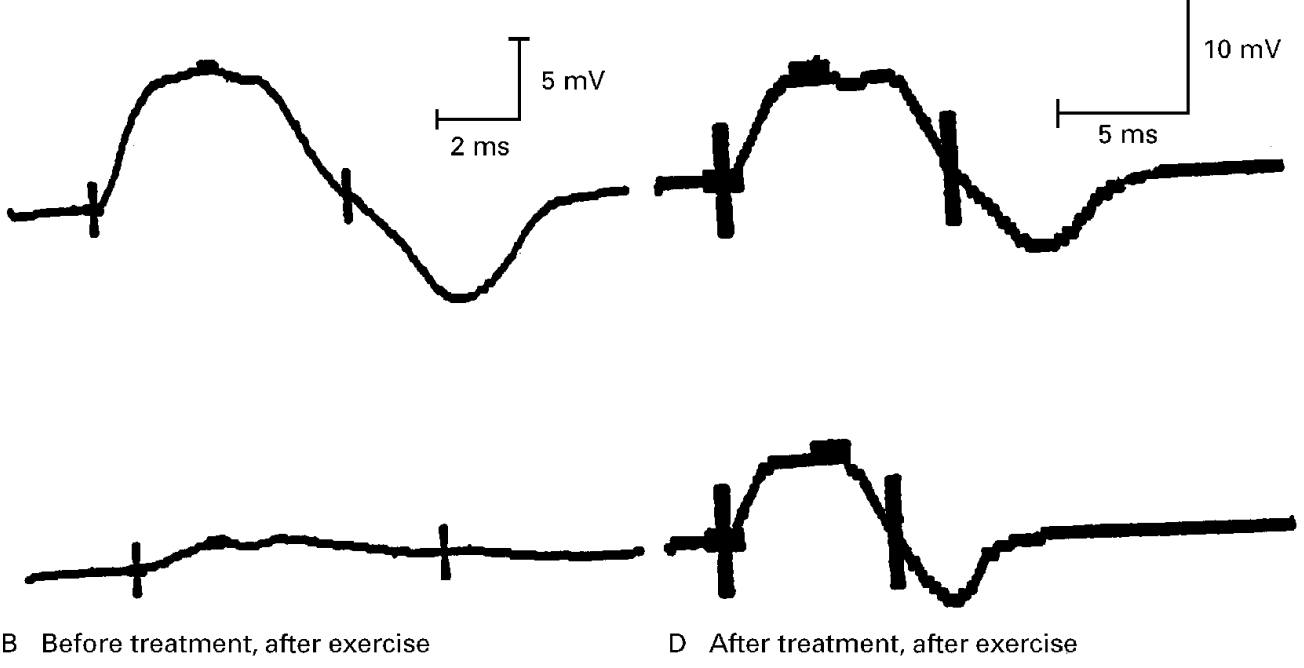

B Before treatment, after exercise

D After treatment, after exercise

Figure 1 Postexercise decrement in ADM CMAP before and after treatment with $4 \mathrm{mg}$ oral salbutamol.

Routine EMG and nerve conduction studies were normal. There was no evidence of myotonia and cooling did not produce any paramyotonia. She underwent a standard exercise test as previously described by McManis et $a l^{4}$ for the detection of periodic paralysis. This involves measuring the compound muscle action potential (CMAP) from the abductor digiti minimi muscle before and for up to two hours after a five minute period of sustained exercise with brief rests. After this protocol a progressive decline in CMAP amplitude was found with a maximum decrement of $85 \%$ at one hour. A decrement $>40 \%$ is considered to be a positive result. ${ }^{4}$ Repeat testing one hour after an oral dose of $4 \mathrm{mg}$ salbutamol showed a pronounced improvement; on this occasion the maximum CMAP decrement had normalised to only $17.5 \%$ (fig 1 ).

Total DNA was extracted from blood. Exon 13 of the SCN4A gene was analysed by direct sequence analysis of both DNA strands using an automated DNA sequencer (primer sequences are available from the authors on request). A heterozygous $\mathrm{C}$ to $\mathrm{T}$ point mutation at nucleotide position 2188 was identified. This mutation predicts a threonine to methionine substitution at amino acid position 704 in segment 5 of the second repeated domain of the $\alpha$-subunit of the skeletal muscle sodium channel. This point mutation associates with hyperkalaemic periodic paralysis. ${ }^{12}$

The patient has been supplied with a salbutamol metered dose inhaler and has been able to abort her attacks successfully with two 100 mcg metered inhalations.

Prophylaxis with salbutamol has not proved necessary.

\section{Discussion}

At least 19 different disease associated point mutations have now been identified in the SCN4A. ${ }^{5}$ Five of these associate with hyperkalaemic periodic paralysis, whereas the remainder associate either with paramyotonia congenita or with one of the potassium aggravated myotonias. ${ }^{5}$ The thr 704 met mutation, identi- fied in the case reported here, is the most common cause of hyperkalaemic periodic paralysis. Some patients harbouring this mutation also exhibit generalised myotonia, which was not present in our case. Most of the reported thr704met cases develop a vacuolar myopathy in later life which can be significantly disabling. ${ }^{5}$ It seems likely that this was the cause of the patient's mother being wheelchair bound. The myopathy tends to develop in later years when the attacks of paralysis decline or subside altogether. The precise pathogenesis of the myopathy is undetermined and it does not seem to relate to the frequency or severity of the paralytic attacks. ${ }^{1}$ It is not known whether effective therapy for the attacks reduces the likelihood of developing a myopathy later. ${ }^{1}$ It is also unclear why the thr704met mutation should particularly associate with a fixed myopathy whereas the other four point mutations do not. Electrophysiological studies of the 704 mutation expressed in cell systems have shown that the biophysical defect induced by this mutation is a failure of sodium channel fast inactivation, ${ }^{5}$ leading to a persistent inward sodium current. It is possible that this increased "ionic traffic" may be relevant to the development of the vacuolar myopathy.

The identification of mutations responsible for hyperkalaemic periodic paralysis makes DNA based diagnosis now possible. This will allow precise genotype-phenotype correlations to be made and the assessment of whether the therapeutic response varies with different mutations. Therapy for hyperkalaemic periodic paralysis comprises avoiding known precipitants in combination with acute or prophylactic medication. The most commonly used agents are thiazide diuretics or carbonic anhydrase inhibitors such as acetazolamide. Both of these agents lower serum potassium. However, they are not always effective and $\beta$-adrenergic agents have previously been suggested to be of benefit. ${ }^{67}$ Their mode of action is likely to be stimulation of the sodium potassium pump tending to lower serum potassium and reducing the sodium influx into the muscle fibre. ${ }^{8}$ 
Salbutamol proved to be very effective in the present case and we were able to document an electrophysiological response which has not been reported previously. It remains to be seen whether different amino acid substitutions in the protein encoded by SCN4A predict the therapeutic response to different agents.

We conclude that therapy with salbutamol should be considered in patients with hyperkalaemic periodic paralysis with the 704 mutation, either as a first line agent or when other treatment options have failed. This study indicates that electrophysiological studies are a useful means of quantitifying and monitoring response to therapy as well as a diagnostic aid.

We thank Dr JW Scadding for referring this patient and Dr L Ptacek for providing positive control samples for sodium chan- nel gene point mutations. Financial support from the Brain Research Trust is gratefully acknowledged.

1 Lehmann-Horn F, Engel AG, Ricker K, et al. The periodic paralyses and paramyotonia congenita. In: Engel AG, Franzini-Armstrong C, eds. Myo
Hill, New York, 1994:1303-402.

2 Ptacek LJ, George AL, Griggs RC, et al. Identification of a mutation in the gene causing hyperkalaemic periodic paralysis. Cell 1991;67:1021-7.

3 Subramony SH, Wee AS. Exercise and rest in hyperkalaemic periodic paralysis. Neurology 1986;36:173.

4 McManis PG, Lambert EH, Jasper RD. The exercise test in periodic paralysis. Muscle Nerve 1986;9:704-10.

5 Lehmann-Horn, Rudel R. Molecular pathophysiology of voltage-gated ion channels. Rev Physiol Biochem Pharmacol 1996;128:196-268.

6 Wang P, Clusen T. Treatment of hyperkalaemic periodic Wang P, Clusen T. Treatment of hyperkalaemic periodic
paralysis by inhalation of salbutamol. Lancet $1976 ; \mathrm{i}: 221$.

7 Benheim PE, Reale EO, Berg BO. $\beta$-Adrenergic treatment of hyperkalaemic periodic paralysis. Neurology 1985;35: 746.

8 Clausen T. Regulation of active $\mathrm{Na}^{+}-\mathrm{K}^{+}$transport in skeletal muscle. Physiol Rev 1986;66:542. 